\title{
Nano-molar level determination of isoprenaline in pharmaceutical and clinical samples; a nanostructure electroanalytical strategy
}

\author{
Sareh Sadat Moshirian-Farahia ${ }^{\text {|Hassan Ali Zamania,* }}{ }^{\circledR}$ |Mohamadreza Abedib
}

aDepartment of Applied Chemistry, A high sensitive electroanalytical-based sensor for Mashhad Branch, Islamic Azad determination of isoprenaline was fabricated by modification of University, Mashhad, Iran

${ }^{b}$ Department of Applied Chemistry, Quchan Branch, Islamic Azad University, Quchan, Iran carbon paste electrode (CPE) by NiO-Pt-Pd/CNTs composite as conductive mediator and n-hexyl-3-methylimidazolium hexafluoro phosphate (NHIHP) as conductive binder. The NHIHP/NiO-Pt-Pd/CNTs/CPE was improved the oxidation signal of isoprenaline $\square 3.47$ times and reduced oxidation overpotential of drug $\square 180 \mathrm{mV}$. The $\mathrm{pH}$ investigation confirmed that redox behavior of isoprenaline is depended of $\mathrm{pH}$ solution with equal value of electron and proton in redox mechanism. The NHIHP/NiO-Pt-Pd/CNTs/CPE was successfully used for determination of isoprenaline in the concertation range 0.003$300 \mu \mathrm{M}$ with detection limit $0.9 \mathrm{nM}$ by square wave voltammetric method. The standard addition results showed powerful ability of NHIHP/NiO-Pt-Pd/CNTs/CPE as an electroanalytical tool for determination of isoprenaline in the pharmaceutical and clinical samples with recovery data 98.76$105.06 \%$.

*Corresponding Author:

Hassan Ali Zamani

\section{KEYWORDS}

Email:haszamani@yahoo.com

Tel.: +98 (51) 36630781

Isoprenaline; NiO-based composite; nanostructure sensor; modified carbon paste electrode; drug sensor.

\section{Introduction}

Isoprenaline is one of the famous catecholamine drugs that prescribed for treatment of heart block and bradycardia. In addition, the treat isoprenaline prescribed for treat asthma and is not suggest for patients with tachyarrhythmias [1]. Although isoprenaline is an active and useful drug in premature infants, a few published papers described this point [2]. The nervousness, visual blurring, headache, nausea and dizziness are the most important side effect associated with overdose of isoprenaline. Therefore, the fast detection and sensitive determination of isoprenaline in biological and clinical sample are very important and many analytical methods were suggested for this goal in recent years [3-6].

The tendency to use electrochemical sensors for the analysis of pharmaceutical compounds such as isoprenaline has grown significantly in recent years due to the high rate of analysis and the lack of use of toxic solvents [7-19]. With the advent of modified sensors and greater variability in the manufacture of electrochemical sensors, this technique has been introduced as a serious competitor for chromatographic methods in drug composition analysis [20-30]. The use of nanomaterial-modified sensors has been a major revolution in the design of sensitive sensors in recent years [31-41]. Nanomaterials with wide range application and new properties can be introduced as 
powerful catalysts in different industries such as electrochemical sensor filed [42-58]. The using nanomaterials and especially metalbased nanoparticle can be improve sensitivity of electrochemical sensors and suggest as a new strategy for design of new type of electroanalytical sensors in drug analysis [5979]. In addition, ionic liquids showed more advantages for modification of electrochemical sensors due to high electrical conductivity and wide electrochemical windows range [80-83]. Recently, the many of scientific reports showed high performance ability of ionic liquid/nanomaterial composite for modification of electrochemical sensor with highly sensitive properties [84-86].

In this research, we fabricated NHIHP/NiO-Pt-Pd/CNTs/CPE as a powerful and highly sensitive electroanalytical sensor for determination of isoprenaline. For this goal, the NiO-Pt-Pd/CNTs composite was synthesized by a simple precipitation method. Due to presence of NiO-Pt-Pd/CNTs nanocomposite and NHIHP as two conductive mediators, the NHIHP/NiO-Pt-Pd/CNTs/CPE showed highly sensitive activity for nanomolar determination of isoprenaline.

\section{Experimental}

\section{Materials and instruments}

Isoprenaline hydrochloride, $98 \%$ was purchased from Across Company. The stock solution of isoprenaline hydrochloride was prepared by dissolving $0.0247 \mathrm{~g}$ isoprenaline hydrochloride powder into $100 \mathrm{~mL}$ distillated water. Graphite powder, diethyl ether and paraffin oil were purchased from Merck Co. and used for preparation of carbon paste electrode. Nickel nitrate hexahydrate, platinum(II) chloride, palladium(II) acetylacetonate, sodium hydroxide and single wall carbon nanotubes were purchased from Sigma-Aldrich Co. and used for synthesis of NiO-Pt-Pd/CNTs nanocomposite. Phosphoric acid was purchased from Across
Co. and used for preparation of phosphate buffer solution (PBS 0.1 M).

Electrochemical instrument (The IviumVertex and Potentiostat/Galvanostat) was used for voltametric investigation. $\mathrm{Ag} / \mathrm{AgCl} / \mathrm{KCl}$ and $\mathrm{Pt}$ wire were purchased from Azar Electrode CO. and used as reference and counter electrodes, respectively.

\section{Synthesis of NiO-Pt-Pd/CNTs nanocomposite}

The $8.0 \mathrm{mg}$ platinum(II) chloride + palladium(II) acetylacetonate was dissolved in $100 \mathrm{~mL}$ solution containing nickel nitrate hexahydrate $(0.5 \mathrm{M})$. The solution was stirred for $30 \mathrm{~min}$ at $35{ }^{\circ} \mathrm{C}$. In next step, $200 \mathrm{~mL}$ sodium hydroxide $1.0 \mathrm{M}$ was added to solution.The precipitate was washed seven times and then filtered. The filtered precipitate was dried at $2{ }^{\circ} \mathrm{C}$ for $2 \mathrm{~h}$ and then calcined in the furnace at $2{ }^{\circ} \mathrm{C}$.

\section{Preparation of NHIHP/NiO-Pt-Pd/CNTs/CPE}

The NHIHP/NiO-Pt-Pd/CNTs/CPE was ready by mixing $0.95 \mathrm{gr}$ graphite powder $+0.05 \mathrm{~g}$ Pt- NiO-Pt-Pd/CNTs +0.95 gr graphite powder into mortar and pestle in the presence of diethyl ether as solvent for better mixing. After the evaporation of diethyl ether in the room temperature, 1 drops of NHIHP and 14 drops of paraffin oil were added to the dried powders as binders handmixed for $3.0 \mathrm{~h}$. A homogeneous paste was obtained and then filled into a glass tube and it was attached to an electrical contact (copper wire).

\section{Preparation of real sample}

Isoprenaline injection was purchased from local pharmacy and then mix with PBS $(\mathrm{pH}=7.0)$. The injection solution of isoprenaline with specified concentration was used for testing of NHIHP/NiO-Pt$\mathrm{Pd} / \mathrm{CNTs} / \mathrm{CPE}$ ability for real sample using standard addition method. In addition, the 
pharmaceutical serum was dilute 5-times with PBS and used for real sample analysis.

\section{Results and discussion}

$\begin{aligned} & \text { Characterization } \\ & \text { nanocomposite }\end{aligned}$

The NiO-Pt-Pd/CNTs nanocomposite was characterized by FESEM and EDS methods.

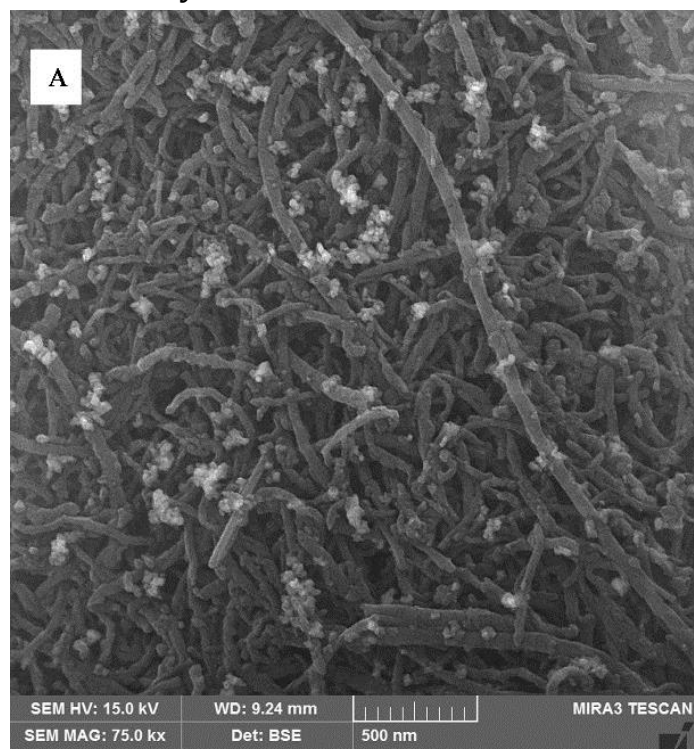

The results in Figure $1 \mathrm{~A}$ confirm spherical NiO-Pt-Pd deposit as surface of single wall carbon nanotubes. In addition, the analysis results relative to EDS investigation are presence in figure $2 \mathrm{~B}$ and presence of $\mathrm{C}, \mathrm{Ni}, \mathrm{O}$, $\mathrm{Pt}$ and Pt confirm purity of synthesized NiOPt-Pd/CNTs nanocomposite.

B

\begin{tabular}{c|c|}
\hline Element & $\mathrm{Wt} \%$ \\
\hline $\mathrm{C}$ & 40.6 \\
\hline $\mathrm{Ni}$ & 29.2 \\
\hline 0 & 18.1 \\
\hline $\mathrm{Pt}$ & 5.8 \\
\hline $\mathrm{Pd}$ & 6.3 \\
\hline
\end{tabular}

FIGURE 1 A) FESEM and B) EDS analysis data for synthesized NiO-Pt-Pd/CNTs nanocomposite

\section{Electrochemical investigation}

Isoprenaline is a catecholamine drug and its electrochemical behavior is expected to be influenced by $\mathrm{pH}$ of aqueous solutions. Therefore, this factor was optimized by recording cyclic voltammograms of $600 \mu \mathrm{M}$ isoprenaline in the $\mathrm{pH}$ range 4.0-8.0 (Figure 2 inset). The maximum oxidation current was observe at $\mathrm{pH}=7.0$ and this value was selected as optimum condition. The linear relation with equation of $E_{p a}=0.0592 \mathrm{pH}+0.9167$ $\left(\mathrm{R}^{2}=0.9902\right)$ was observe between oxidation potential of isoprenaline and $\mathrm{pH}$ of solution in the presence of drug that confirm equal value of electron and proton in redox behavior of isoprenaline (Figure 2).

The cyclic voltammograms of $600 \mu \mathrm{M}$ isoprenaline was recorded at surface of NHIHP/NiO-Pt-Pd/CNTs/CPE (Figure 3 curve a), NHIHP/CPE (Figure 3 curve b), NiO-Pt$\mathrm{Pd} / \mathrm{CNTs} / \mathrm{CPE}$ (Figure 3 curve c) and NHIHP/NiO-Pt-Pd/CNTs/CPE (Figure 3 curve d) in the solution with $\mathrm{pH}=7.0$. With moving of CPE to NHIHP/NiO-Pt-Pd/CNTs/CPE, the oxidation current of isoprenaline was increased from $12.8 \mu \mathrm{A}$ to $43.8 \mu \mathrm{A}$ and oxidation potential was reduce from $680 \mathrm{mV}$ to $500 \mathrm{mV}$. 


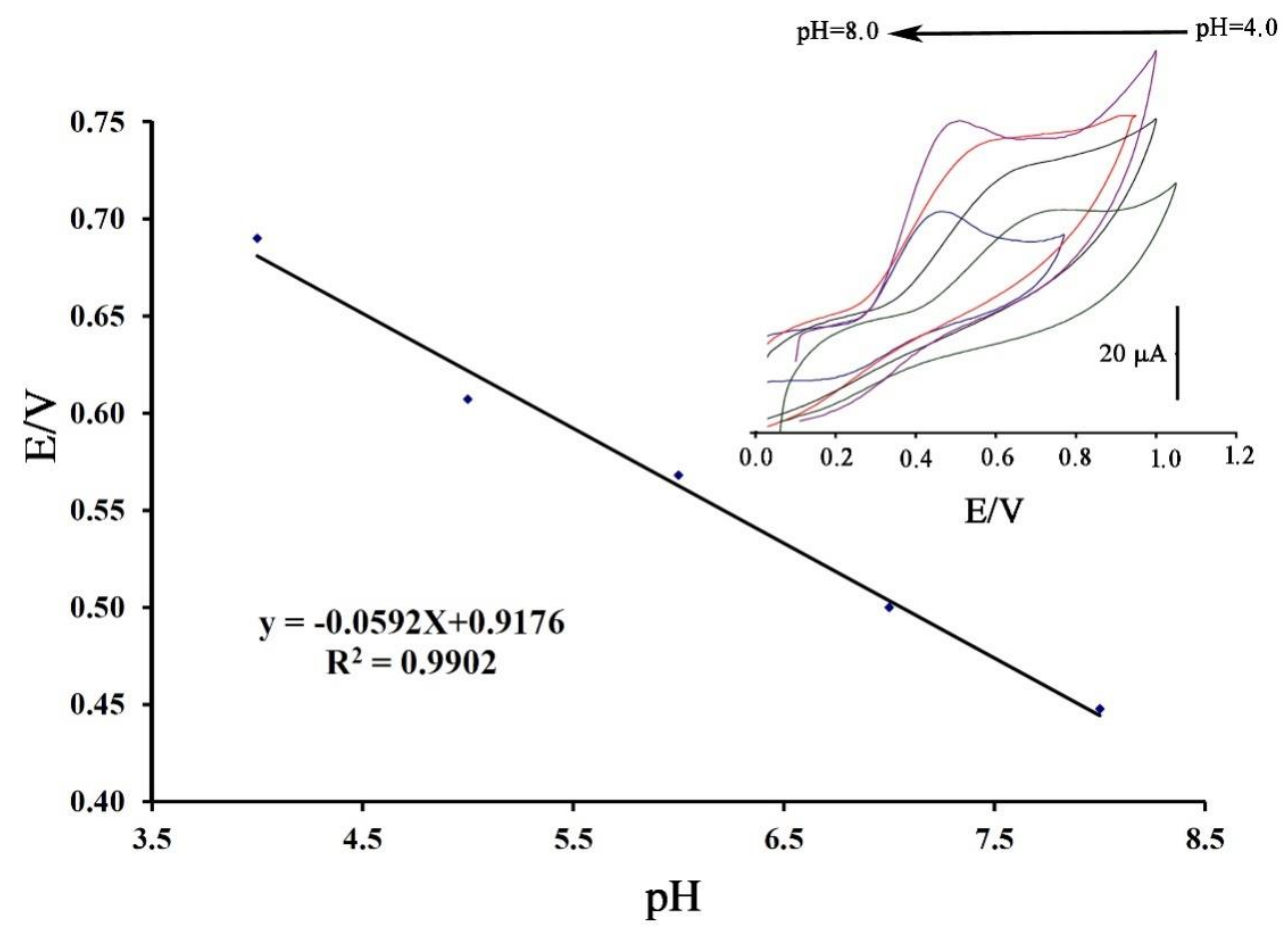

FIGURE 2 Diagram of $E_{p} v s$. pH for electro-oxidation of $600 \mu \mathrm{M}$ isoprenaline at surface of NHIHP/NiO-Pt-Pd/CNTs/CPE. Insert) Cyclic voltammogram relative to electro-oxidation of 600 $\mu \mathrm{M}$ isoprenaline at surface of NHIHP/NiO-Pt-Pd/CNTs/CPE in the $\mathrm{pH}$ range 4.0-8.0.

This improvement is relative to presence of NHIHP and NiO-Pt-Pd/CNTs at surface of $\mathrm{CPE}$ as conductive binders.

The cyclic voltammograms of $550 \mu \mathrm{M}$ isoprenaline was recorded at surface of
NHIHP/NiO-Pt-Pd/CNTs/CPE was recording in the scan rate range $10-100 \mathrm{mV} / \mathrm{s}$ (Figure 4).

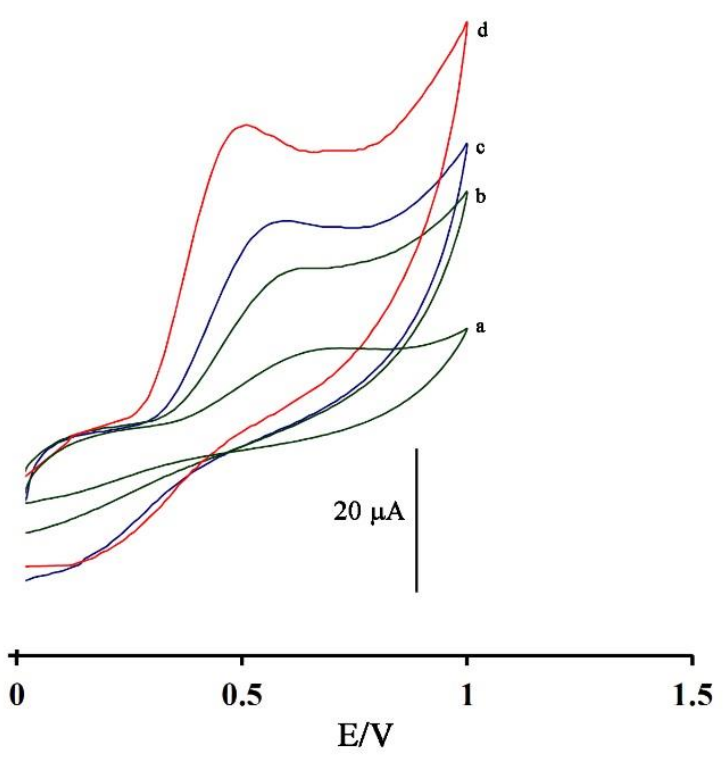

FIGURE 3 Cyclic voltammogram of $600 \mu \mathrm{M}$ isoprenaline at surface of a) CPE, b) NiO-PtPd/CNTs/CPE, c) NHIHP/CPE and d) NHIHP/NiO-Pt-Pd/CNTs/CPE 


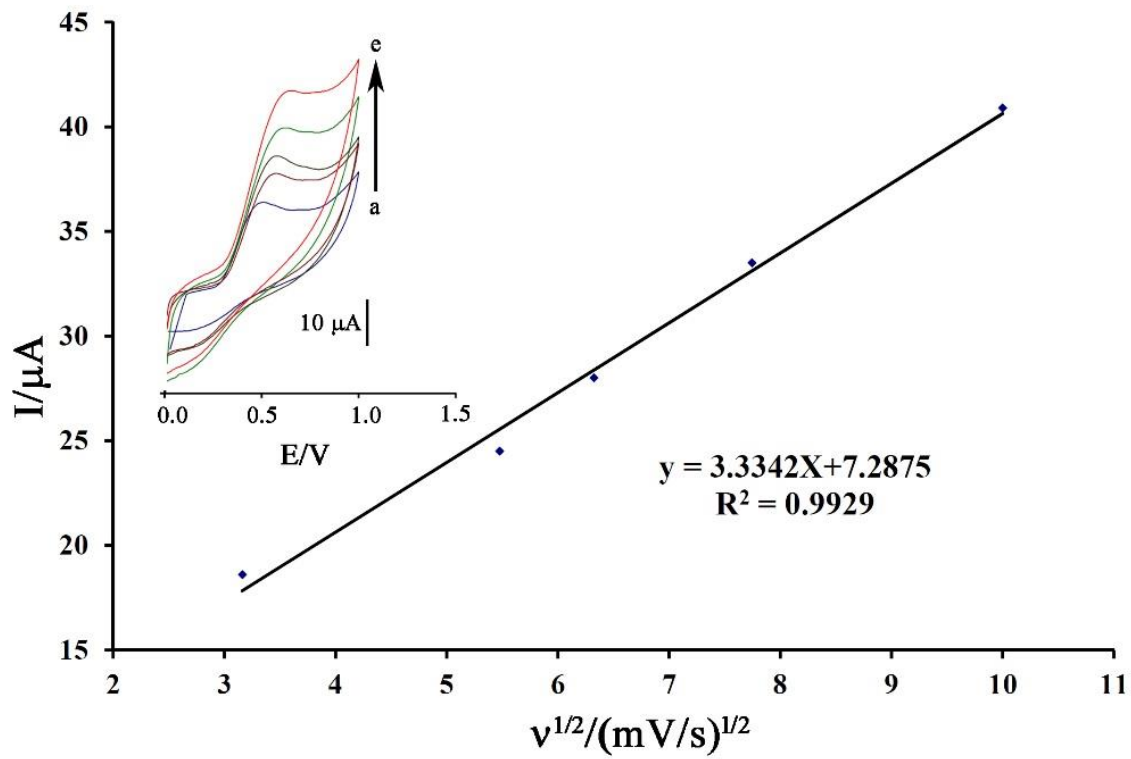

FIGURE 4 Cyclic voltammogram of $550 \mu \mathrm{M}$ isoprenaline at surface of NHIHP/NiO-Pt$\mathrm{Pd} / \mathrm{CNTs} / \mathrm{CPE}$ in the scan rate a) 10, b) 30, c) 40, d) 60 and e) $100 \mathrm{mV} / \mathrm{s}$.

The linear relation between oxidation current of isoprenaline and $v^{1 / 2}$ at surface of NHIHP/NiO-Pt-Pd/CNTs/CPE confirm a diffusion process [87-96] in this study.

Chronoamperometric signals of isoprenaline were recorded at a surface of NHIHP/NiO-Pt-Pd/CNTs/CPE (Figure 5 A).
For this goal, we used step potential at 400 $\mathrm{mV}$. The Plot of I versus $\mathrm{t}^{-1 / 2}$ were recorded for 100,200 and $300 \mu \mathrm{M}$ isoprenaline (Figure 6B). The value of diffusion coefficient (D) was obtained $\sim 2.14 \times 10^{-6} \mathrm{~cm}^{2} / \mathrm{s}$ by the Cottrell equation.

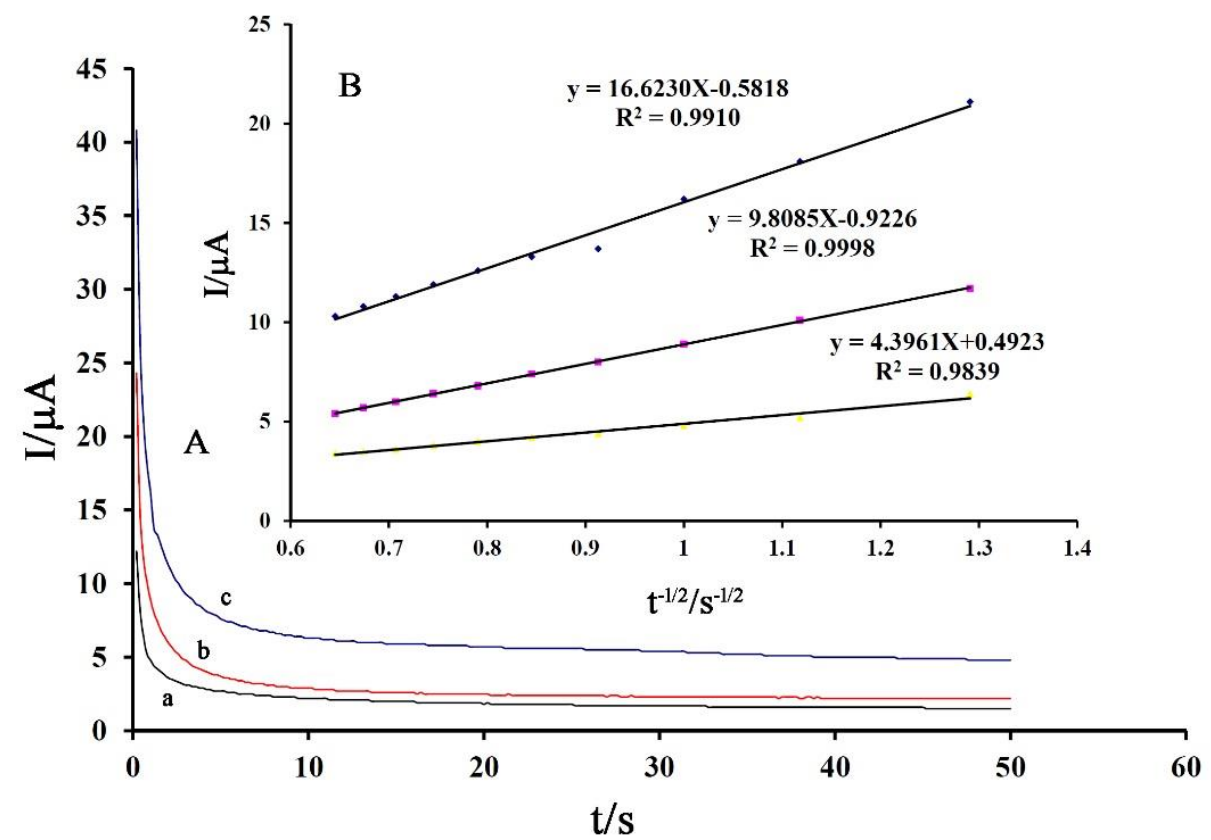

FIGURE 5 A) Chronoamperograms (a) 100.0, (b) 200.0 and (c) $300.0 \mu \mathrm{M}$ isoprenaline at the NHIHP/NiO-Pt-Pd/CNTs/CPE. B) Cottrell plots obtained from chronoamperometry 
The square wave voltammograms of isoprenaline (in the different concentration range) was recorded at surface of NHIHP/NiO-Pt-Pd/CNTs/CPE (Figure 7 inset). The results show a linear dynamic range $\quad 0.003-300 \mu \mathrm{M} \quad\left(\mathrm{R}^{2}=0.9918\right) \quad$ and detection limit $0.9 \mathrm{nM}$ that confirm powerful ability of NHIHP/NiO-Pt-Pd/CNTs/CPE as an electroanalytical sensor in determination trace level of isoprenaline.

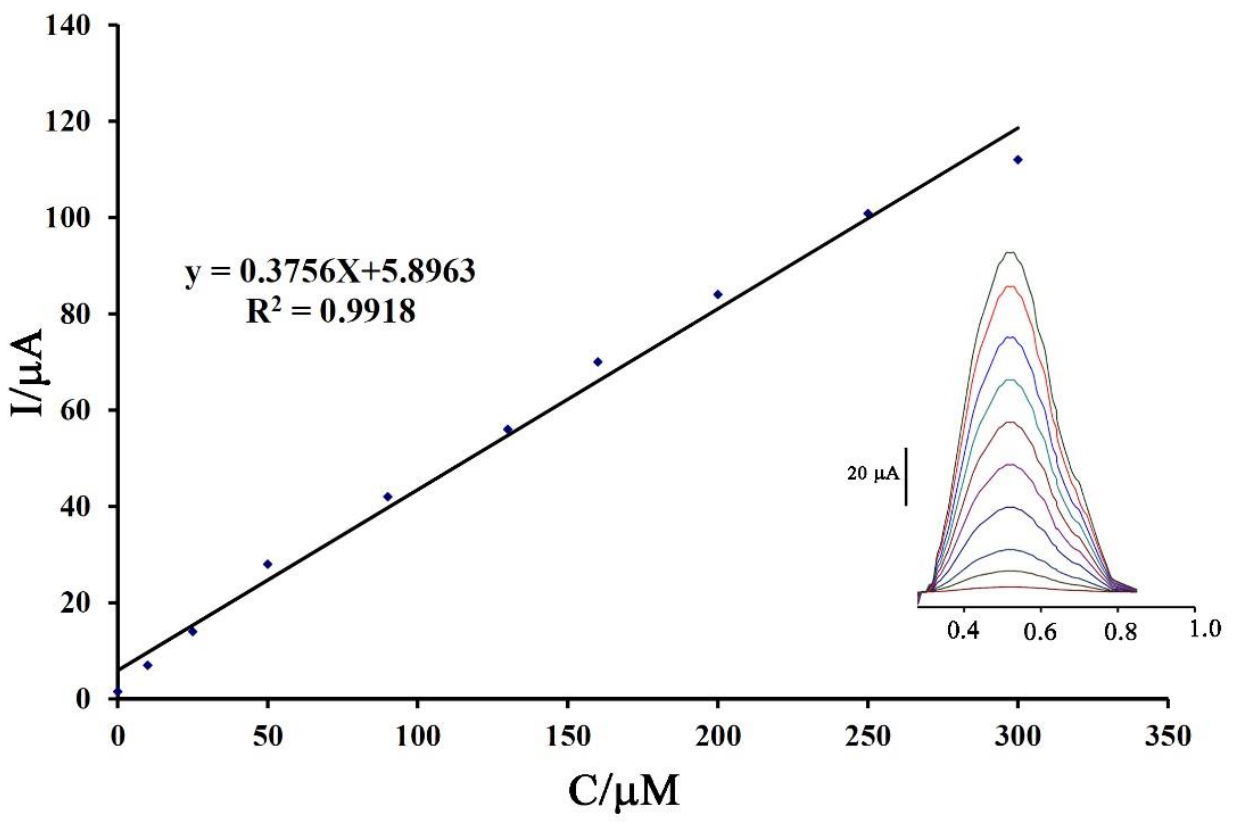

FIGURE 7 Relation between oxidation current of isoprenaline and its concentration in the linear dynamic range 0.003-300 $\mu \mathrm{M}$ at surface of NHIHP/NiO-Pt-Pd/CNTs/CPE. Insert) Relative square wave voltammograms

The stability of NHIHP/NiO-Pt$\mathrm{Pd} / \mathrm{CNTs} / \mathrm{CPE}$ was evaluated over a period of 30 days in the presence $600 \mu \mathrm{M}$ isoprenaline. The results showed $92.12 \%$ of initial signal of isoprenaline was remain after 30 days that confirm good stability of NHIHP/NiO-Pt$\mathrm{Pd} / \mathrm{CNTs} / \mathrm{CPE}$.

The selectivity of NHIHP/NiO-Pt$\mathrm{Pd} / \mathrm{CNTs} / \mathrm{CPE}$ for determination of $20.0 \mu \mathrm{M}$ isoprenaline was investigated using square wave voltammetric method with acceptable error $5 \%$ in current. The results showed that 500 -fold of glucose, methanol, 800-fold of $\mathrm{Mg}^{2+}, \mathrm{F}^{-}$and $\mathrm{K}^{+}$and 400 -fold of valine, methionine, vitamin $\mathrm{B}_{9}$ and tryptophan and 20 -fold of dopamine and vitamin $\mathrm{B}_{2}$ did not affect the selectivity.

The capability of NHIHP/NiO-Pt$\mathrm{Pd} / \mathrm{CNTs} / \mathrm{CPE}$ for determination of isoprenaline in injection, and pharmaceutical serum samples (as real samples) was checked. The obtained data are presence in table 1 and recovery data 98.76-105.06\% confirm powerful ability of NHIHP/NiO-Pt$\mathrm{Pd} / \mathrm{CNTs} / \mathrm{CPE}$ as a new and powerful electroanalytical sensor in determination of isoprenaline.

TABLE 1 Determination of isoprenaline in real samples $(n=5)$.

\begin{tabular}{ccccc}
\hline Samples & $\begin{array}{c}\text { Added } \\
(\boldsymbol{\mu M})\end{array}$ & $\begin{array}{c}\text { Expected } \\
(\boldsymbol{\mu M})\end{array}$ & $\begin{array}{c}\text { Founded } \\
(\boldsymbol{\mu M})\end{array}$ & $\begin{array}{c}\text { Recovery } \\
\%\end{array}$ \\
\hline Injection samples & --- & --- & $2.11 \pm 0.22$ & --- \\
Pharmaceutical serum & 10.00 & $11.96 \pm 0.67$ & $10.16 \pm 0.23$ & 98.76 \\
& --- & $<$ LOD & $<$ LOD & -- \\
& 15.00 & 15.00 & $15.76 \pm 0.92$ & 105.06 \\
\hline
\end{tabular}




\section{Conclusion}

The NiO-Pt-Pd/CNTs nanocomposite was synthesis by a simple and fast precipitation method and characterized by FESEM and EDS methods. In continuous the synthesized nanocomposite was used for fabrication of NHIHP/NiO-Pt-Pd/CNTs/CPE. The NHIHP/NiO-Pt-Pd/CNTs/CPE was used as a new electroanalytical sensor for determination of isoprenaline. The NHIHP/NiO-Pt-Pd/CNTs/CPE was successfully used for determination of isoprenaline in the concertation range 0.003$300 \mu \mathrm{M}$ with detection limit $0.9 \mathrm{nM}$ in real sample with recovery data 98.76-105.06\%.

\section{Orcid:}

Hassan Ali Zamani: https://orcid.org/00000002-3156-2781

\section{Acknowledgments}

The authors wish to thank Mashhad Branch, Islamic Azad University for support of this work.

\section{References}

[1] D.C. Plumb, Plumb's Veterinary Drug Handbook: Pocket, John Wiley \& Sons, 2018.

[2] A.M. Lands, F.P. Luduena, H.J. Buzzo, Life Sci., 1967, 6, 2241-2249.

[3] K.O. Lupetti, I.C. Vieira, O. Fatibello-Filho, Talanta., 2002, 57, 135-143.

[4] A. Wong, A.M. Santos, T.A. Silva, O. Fatibello-Filho, Talanta, 2018, 183, 329-338.

[5] A. Kutluay, M. Aslanoglu, J. Acta Chim. Slovenica., 2010, 57, 157-162.

[6] M. Fouladgar, J. Electrochem. Soc., 2016, 163, B38-B42.

[7] V.G. Bonifácio, L.H. Marcolino Jr, M.F. Teixeira, 0. Fatibello-Filho, Microchem. J., 2004, 78, 55-59.

[8] M. Chen, X. Ma, X. Li, J. Solid State Electr., 2012, 16, 3261-3266.

[9] M. Baghayeri, A. Amiri, B. Maleki, Z. Alizadeh, O. Reiser, Sens. Actuators B., 2018, 273, 1442-1450.
[10] S. Aftab, G. Ozcelikay, S. Kurbanoglu, A. Shah, F.J. Iftikhar, S.A. Ozkan, J. Pharmaceut. Biomed. Anal., 2019, 165, 73-81.

[11] F. Faridbod, A.L. Sanati, Curr. Anal. Chem., 2019, 15, 103-123.

[12] J. Mohanraj, D. Durgalakshmi, R.A. Rakkesh, S. Balakumar, S. Rajendran, H. Karimi-Maleh, J. Colloid Interf. Sci., 2020, https://doi.org/10.1016/j.jcis.2020.01.089.

[13] S. Kaya, B. Demirkan, N. Bakirhan, E. Kuyuldar, S. Kurbanoglu, S. Ozkan, F. Sen, J. Pharmaceut. Biomed. Anal., 2019, 174, 206213.

[14] H. Karimi-Maleh, F. Karimi, M. Alizadeh, A.L. Sanati, Chem. Rec., 2020, 20, https://doi.org/10.1002/tcr.201900092

[15] H. Veisi, F.H. Eshbala, S. Hemmati, M. Baghayeri, RSC Adv., 2015, 5, 10152-10158.

[16] M. Baghayeri, A. Sedrpoushan, A. Mohammadi, M. Heidari, Ionics., 2017, 23, 1553-1562

[17] T. Eren, N. Atar, M.L. Yola, H. KarimiMaleh, Food Chem., 2015, 185, 430-436

[18] M. Fouladgar, Sensor Actuat. B., 2016, 230, 456-462.

[19] W.H. Elobeid, A.A. Elbashir, Prog. Chem. Biochem. Res., 2019, 2, 24-33.

[20] M. Eldefrawy, E.G.A. Gomaa, S. Salem, F. Abdel Razik, Prog. Chem. Biochem. Res., 2018, 1, 11-18.

[21] H. Pyman, H. Roshanfekr, S. Ansari, Eurasian Chem. Commun., 2020, 2, 213-225.

[22] S. Ershad, R. Mofidi Rasi, Eurasian Chem. Commun., 2019, 1, 43-52.

[23] M. Baghayeri, A. Amiri, Z. Alizadeh, H. Veisi, E. Hasheminejad, J. Electroanal. Chem., 2018, 810, 69-77

[24] A. Khodadadi, E. Faghih-Mirzaei, H. Karimi-Maleh, A. Abbaspourrad, S. Agarwal, V.K. Gupta, Sensor. Actuat. B, 2019, 284, 568574.

[25] M. Miraki, H. Karimi-Maleh, M.A. Taher, S. Cheraghi, F. Karimi, S. Agarwal, V.K. Gupta, J. Mol. Liq., 2019, 278, 672-676.

[26] H. Karimi-Maleh, M. Sheikhshoaie, I. Sheikhshoaie, M. Ranjbar, J. Alizadeh, N.W. Maxakato, New J. Chem., 2019, 43, 2362-2367. 
[27] M Baghayeri, M. Rouhi, M.M. Lakouraj, M. Amiri-Aref, J. Electroanal. Chem., 2017, 784, 69-76.

[28] A.L. Sanati, F. Faridbod, M.R. Ganjali, J. Mol. Liq., 2017, 241, 316-320.

[29] B. Demirkan, S. Bozkurt, A. Şavk, K. Cellat, F. Gülbağca, M.S. Nas, M.H. Alma, F. Sen, Sci. Rep., 2019, 9, Article number: 12258

[30] M. Shamsipur, Z. Karimi, M.A. Tabrizi, S. Rostamnia, J. Electroanal. Chem., 2017, 799, 406-412.

[31] Y. Akbarian, M. Shabani-Nooshabadi, H. Karimi-Maleh, Sensor. Actuat. B., 2018, 273, 228-233.

[32] M. Baghayeri, B. Maleki, R. Zarghani, Mater. Sci. Eng. C., 2014, 44, 175-182

[33] A. Baghizadeh, H. Karimi-Maleh, Z. Khoshnama, A. Hassankhani, M. Abbasghorbani, Food Anal. Methods., 2015, 8, 549-557.

[34] M. Bijad, H. Karimi-Maleh, M. Farsi, S.-A. Shahidi, J. Food Meas. Charact., 2018, 12, 634640.

[35] S.A. Alavi-Tabari, M.A. Khalilzadeh, H. Karimi-Maleh, J. Electroanal. Chem., 2018, 811, 84-88.

[36] M. Shabani-Nooshabadi, F. TahernejadJavazmi, RSC Adv., 2015, 5, 56255-56261.

[37] A.N. Golikand, J. Raoof, M. Baghayeri, M. Asgari, L. Irannejad, Russ. J. Electrochem., 2009, 45, 192-198.

[38] M. Shabani-Nooshabadi, F. Tahernejad-Javazmi, Electroanalysis., 2015, 27, 1733-1741.

[39] Y. Sheng, W. Qian, J. Huang, B. Wu, J. Yang, T. Xue, Y. Ge, Y. Wen, Microchim. Acta., 2019, 186, 543.

[40] X. Wu, W. Zhang, C. Morales-Verdejo, Y. Sheng, M.B. Camarada, L. Chen, Z. Huang, Y. Wen, J. Electroanal. Chem., 2019, 839, 177186.

[41] T. Zabihpour, S.-A. Shahidi, H. Karimi Maleh, A. Ghorbani-Hasan Saraei, Eurasian Chem. Commun., 2020, 2, 362-373.

[42] S. Malekmohammadi, H. Hadadzadeh, S. Rezakhani, Z. Amirghofran, ACS Biomater. Sci. Eng., 2019, 5, 4405-4415.
[43] S. Malekmohammadi, H. Hadadzadeh, Z. Amirghofran, J. Mol. Liq., 2018, 265, 797-806. [44] S. Rayati, S. Malekmohammadi, J. Exp. Nanosci., 2016, 11, 872-883.

[45] E. Hosseini, A. Rajaei, M. Tabatabaei, A. Mohsenifar, K. Jahanbin, Food Biophys., 2019. https://doi.org/10.1007/s11483-019-09612$\mathrm{z}$

[46] M. Dehhaghi, M. Tabatabaei, M. Aghbashlo, H.K.S. Panahi, A.-S. Nizami, J. Environ. Manage., 2019, 251, 109597.

[47] H. Hosseinzadeh-Bandbafha, E. Khalife, M. Tabatabaei, M. Aghbashlo, M. Khanali, P. Mohammadi, T.R. Shojaei, S. Soltanian, Energ. Convers. Manage., 2019, 196, 1153-1166.

[48] A. Razmjou, G. Eshaghi, Y. Orooji, E. Hosseini, A.H. Korayem, F. Mohagheghian, Y. Boroumand, A. Noorbakhsh, M. Asadnia, V. Chen, Water Res., 2019, 159, 313-323.

[49] M. Aghababaie, M. Beheshti, A. Razmjou, A.-K. Bordbar, Biofuels., 2020, 11, 93-99.

[50] A. Razmjou, M. Asadnia, E. Hosseini, A.H. Korayem, V. Chen, Nat. Commun., 2019, 10, 115.

[51] W. Cha-Umpong, E. Hosseini, A. Razmjou, M. Zakertabrizi, A.H. Korayem, V. Chen, J. Membrane Sci., 2019, 117687.

[52] Y. Orooji, A.a. Alizadeh, E. Ghasali, M.R. Derakhshandeh, M. Alizadeh, M.S. Asl, T. Ebadzadeh, Ceram. Int., 2019, 45, 2084420854.

[53] Y. Orooji, E. Ghasali, M. Moradi, M.R. Derakhshandeh, M. Alizadeh, M.S. Asl, T. Ebadzadeh, Ceram. Int., 2019, 45, 1628816296.

[54] Y. Orooji, M.R. Derakhshandeh, E. Ghasali, M. Alizadeh, M.S. Asl, T. Ebadzadeh, Ceram. Int., 2019, 45, 16015-16021.

[55] H. Karimi-Maleh, M. Shafieizadeh, M.A. Taher, F. Opoku, E.M. Kiarii, P.P. Govender, S. Ranjbari, M. Rezapour, Y. Orooji, J. Mol. Liq., 2019, 298, https://doi.org/10.1016/j.molliq.2019.11204 0 .

[56] T. Jamali, H. Karimi-Maleh, M.A. Khalilzadeh, LWT-Food Sci. Technol., 2014, $57,679-685$. 
[57] E. Doustkhah, M. Heydarizadeh, Z. Fathi, H. Mohtasham, S. Rostamnia, M. Hasani, Chem. Methodol., 2019, 3, 626-634.

[58] E. Doustkhah, Y. Ide, Bursting ACS Appl. Nano Mater., 2019, 2,7513-7520

[59] F. Tahernejad-Javazmi, M. ShabaniNooshabadi, J. Electrochem. Soc., 2017, 164, H975-H980.

[60] M. Beytur, F. Kardaş, O. Akyıldırım, A. Özkan, B. Bankoğlu, H. Yüksek, M.L. Yola, N. Atar, J. Mol. Liq., 2018, 251, 212-217.

[61] S. Salmanpour, A. Sadrnia, F. Karimi, N. Majani, M.L. Yola, V.K. Gupta, J. Mol. Liq., 2018, 254, 255-259.

[62] V.K. Gupta, H. Mahmoody, F. Karimi, S. Agarwal, M. Abbasghorbani, Int. J. Electrochem. Sci., 2017, 12, 248-257.

[63] M. Keyvanfard, M. Ahmadi, F. Karimi, K. Alizad, Chin. Chem. Lett., 2014, 25, 12441246.

[64] F. Tahernejad-Javazmi, M. ShabaniNooshabadi, H. Karimi-Maleh, Talanta., 2018, 176, 208-213.

[65] S. Cheraghi, M.A. Taher, H. Karimi-Maleh, J. Food Comp. Anal., 2017, 62, 254-259.

[66] M. Bijad, H. Karimi-Maleh, M.A. Khalilzadeh, Food Anal. Methods., 2013, 6, 1639-1647.

[67] M. Najafi, M.A. Khalilzadeh, H. KarimiMaleh, Food Chem., 2014, 158, 125-131.

[68] M. Elyasi, M.A. Khalilzadeh, H. KarimiMaleh, Food Chem., 2013, 141, 4311-4317.

[69] M. Anu Prathap, B. Kaur, R. Srivastava, Chem. Rec., 2019, 19, 883-907.

[70] N.F. Atta, E.H. El-Ads, A. Galal, A.E. Galal, Electroanalysis., 2019, 31, 448-460.

[71] N.F. Atta, A. Galal, S.H. Hassan, J. Electroanal. Chem., 2019, 839, 48-58.

[72] P. Butmee, G. Tumcharern, P. Saejueng, D. Stankovic, A. Ortner, J. Jitcharoen, K. Kalcher, A. Samphao, J. Electroanal. Chem., 2019, 833, 370-379.

[73] M. Govindasamy, S. Shanthi, E. Elaiyappillai, S.-F. Wang, P.M. Johnson, H. Ikeda, Y. Hayakawa, S. Ponnusamy, C. Muthamizhchelvan, Electrochim. Acta., 2019, 293, 328-337.
[74] P.K. Jiwanti, K. Natsui, Y. Einaga, Electrochemistry., 2019, 87, 109-113.

[75] X. Liao, Z. Huang, K. Huang, M. Qiu, F. Chen, Y. Zhang, Y. Wen, J. Chen, J. Electrochem. Soc., 2019, 166, B322-B327.

[76] B. He, H. Liu, Microchem. J., 2019, 150, 104-108.

[77] M. Nurdin, L. Agusu, A.A.M. Putra, M. Maulidiyah, Z. Arham, D. Wibowo, M.Z. Muzakkar, A.A. Umar, J. Phys. Chem. Solids., 2019, 131, 104-110.

[78] L. Wei, H. Cheng, Y. Chen, J. Liu, S. Jia, W. Sun, Int. J. Electrochem. Sci., 2019, 14, 1007010078.

[79] S. Zheng, J. Liu, R. Liu, Z. Hui, J. Tang, X. Wang, Int. J. Electrochem. Sci., 2019, 14, 62346246.

[80] H. Karimi-Maleh, O.A. Arotiba, J. Colloid Interf. Sci., 2020, 560, 208-212.

[81] Z. Shamsadin-Azad, M.A. Taher, S. Cheraghi, H. Karimi-Maleh, J. Food Meas. Charact., 2019, 13, 1781-1787.

[82] H. Karimi-Maleh, C.T. Fakude, N. Mabuba, G.M. Peleyeju, O.A. Arotiba, J. Colloid Interf. Sci., 2019, 554, 603-610.

[83] M. Fouladgar, J. Electrochem. Soc., 2018, 165, B559-B564.

[84] A.L. Sanati, F. Faridbod, Int. J. Electrochem. Sci., 2017, 12, 7997-8005.

[85] M. Shabani-Nooshabadi, M. Roostaee, J. Mol. Liq., 2016, 220, 329-333.

[86] M. Shabani-Nooshabadi, M. Roostaee, F. Tahernejad-Javazmi, J. Mol. Liq., 2016, 219, 142-148.

[87] H. Karimi-Maleh, M.R. Ganjali, P. Norouzi, A. Bananezhad, Mater. Sci. Eng. C., 2017, 73, 472-477.

[88] A.F. Shojaei, K. Tabatabaeian, S. Shakeri, F. Karimi, Sensor. Actuat. B., 2016, 230, 607614.

[89] H. Karimi-Maleh, P. Biparva, M. Hatami, Biosens. Bioelectron., 2013, 48, 270-275.

[90] A.A. Ensafi, H. Karimi-Maleh, J. Electroanal. Chem., 2010, 640, 75-83.

[91] H. Karimi-Maleh, F. Tahernejad-Javazmi, A.A. Ensafi, R. Moradi, S. Mallakpour, H. Beitollahi, Biosens. Bioelectron., 2014, 60, 1-7. 
[92] M.R. Shahmiri, A. Bahari, H. KarimiMaleh, R. Hosseinzadeh, N. Mirnia, Sensor. Actuat. B., 2013, 177, 70-77.

[93] H. Karimi-Maleh, M. Hatami, R. Moradi, M.A. Khalilzadeh, S. Amiri, H. Sadeghifar, Microchim. Acta., 2016, 183, 2957-2964.

[94] H. Karimi-Maleh, A.F. Shojaei, K. Tabatabaeian, F. Karimi, S. Shakeri, R. Moradi, Biosens. Bioelectron., 2016, 86, 879-884.

[95] F. Tahernejad-Javazmi, M. ShabaniNooshabadi, H. Karimi-Maleh, Comp. Part B Eng., 2019, 172, 666-670.
[96] T. Zabihpour, S.A. Shahidi, H. KarimiMaleh, A.G. HasanSaraei, J. Food Meas. Charact., 2020, 14, 1039-1045.

How to cite this article: Sareh Sadat Moshirian-Farahi, Hassan Ali Zamani*, Mohamadreza Abedi. Nano-molar level determination of isoprenaline in pharmaceutical and clinical samples; A nanostructure electroanalytical strategy. Eurasian Chemical Communications, 2020, 2(6), 702-711. Link: http://www.echemcom.com/article_105259. html

Copyright (C) 2020 by SPC (Sami Publishing Company) + is an open access article distributed under the Creative Commons Attribution License, which permits unrestricted use, distribution, and reproduction in any medium, provided the original work is properly cited. 